\title{
Parry Romberg Syndrome: 7 Cases and Literature Review
}

\author{
M. Wong, C.D. Phillips, M. Hagiwara, and D.R. Shatzkes
}

\begin{abstract}
SUMMARY: Parry Romberg syndrome is a rare progressive hemiatrophy of the face that typically occurs in children and young adults and has a peculiar progression that ceases without apparent cause after a highly variable period. Only a subset of patients with Parry Romberg syndrome will develop secondary neurologic or ophthalmologic symptoms, and prognosis is highly variable. Inconsistency in the pattern of atrophy and the development of associated symptoms in patients with Parry Romberg syndrome has made it challenging to diagnose, prognosticate, and treat. The precise etiology of this disease remains unknown, but some authors have implicated sympathetic cervical ganglion dysfunction, abnormal embryogenesis, autoimmune and inflammatory mechanisms, or vasculopathy as potential causes. We present 7 cases of Parry Romberg syndrome and their associated clinical and imaging findings with specific attention to the radiographic characteristics of this disease.
\end{abstract}

ABBREVIATION: PRS = Parry Romberg syndrome

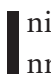
nitially described by Drs Caleb Hillier Parry and Moritiz Heinrich Romberg in 1825 and 1846, respectively, ${ }^{24}$ Parry Romberg syndrome (PRS), also known as progressive facial hemiatrophy, is a rare, insidious, and self-limiting hemiatrophy of the face. It characteristically involves the skin and subcutaneous connective tissues and may later progress to affect the underlying musculature, cartilage, and osseous structures with or without development of neurologic symptoms. ${ }^{1-20,23,26}$ PRS typically presents initially in children and young adults and slowly progresses over a highly variable course ranging from 2 to 20 years, eventually reaching a "burned-out phase" and stabilizing for no apparent reason. $^{2-6,8-10,14-17}$ This peculiar disease course, along with highly variable signs and symptoms, impedes consistent understanding of the underlying pathophysiology of PRS. Many theories about PRS have emerged throughout the years, attributing this syndrome to widely varying etiologies such as infection, trauma, sympathetic nervous system dysfunction, vascular abnormalities, inflammatory conditions, and autoimmune disorders, but at this time, a specific etiology remains uncertain. ${ }^{1-9,13-17,20,25}$

Received July 25, 2014; accepted after revision January 11, 2015.

From the Department of Radiology (M.W., D.R.S.), Lenox Hill Hospital, North Shore Long Island Jewish Health System, New York, New York; Department of Radiology (C.D.P.), Division of Neuroradiology, Weill Medical College of Cornell University, New York Presbyterian Hospital, New York, New York; and Department of Radiology (M.H.), New York University Langone Medical Center, New York, New York.

Please address correspondence to Deborah R. Shatzkes, MD, Lenox Hill Hospital, North Shore Long Island Jewish Health System, Radiology Department, 100 E 77th St, New York, NY 10075; e-mail: dshatzkes@nshs.edu

http://dx.doi.org/10.3174/ajnr.A4297
Changes of cutaneous PRS may be subtle on imaging studies, but $20 \%$ of these patients will have intracranial manifestations that may not correspond to the severity of soft-tissue involvement or neurologic symptoms. ${ }^{13,18,20}$ Radiologic examinations may identify clinically occult intracranial involvement, facilitate the exclusion of other differential diagnostic considerations, ${ }^{15}$ and aid in monitoring disease progression. ${ }^{7}$ We present 7 cases of PRS to illustrate its wide variety of extracranial and intracranial imaging findings, along with a discussion of the clinical course, differential diagnoses, and treatment options. The role of the radiologist in diagnosis and management is emphasized.

\section{Case Series}

Case 1. A 44-year-old woman with known right-sided PRS underwent CT for the evaluation of disease extent and cosmetic surgical planning. CT demonstrated thinning of the skin and subcutaneous fat of the right side of the face extending inferiorly to the suprahyoid neck. There was atrophy of the right platysma and muscles of mastication, with substantial asymmetry in the size of the parotid gland (Fig $1 A$ ). Osseous asymmetry was most pronounced in the maxilla and zygoma, though the right hemimandible was smaller than the left one (Fig 1B). Considerable right enophthalmos was present (Fig 1C). No brain imaging was performed.

Case 2. A 14-year-old boy presented with progressive atrophy of the left side of the face. CT and MR imaging examinations revealed hemiatrophy most prominently involving left-sided skin and subcutaneous fat, with lesser involvement of the mus- 

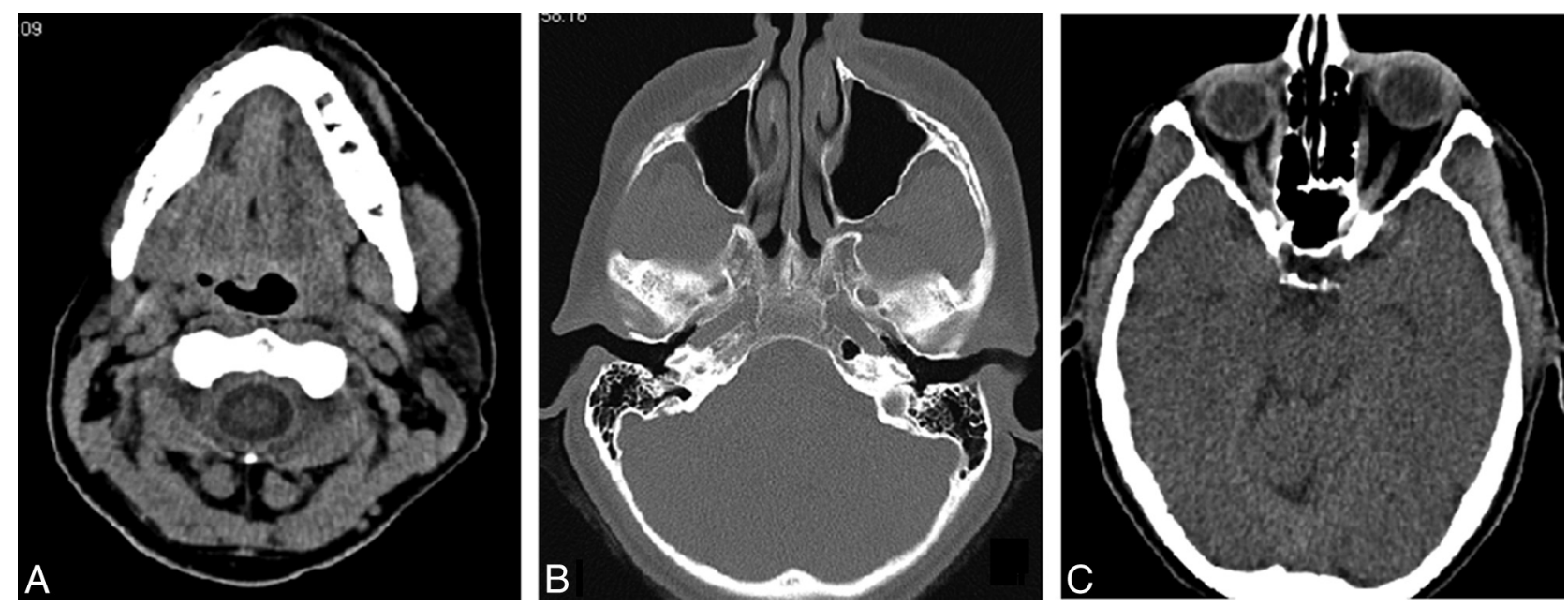

FIG 1. Axial CT images of a 44-year-old woman with PRS obtained for cosmetic surgical planning. $A$, There is prominent hemiatrophy of the skin, subcutaneous fat, and masseter muscle. $B$, The right maxillary sinus is considerably smaller than the left. C, Marked right enophthalmos is present.
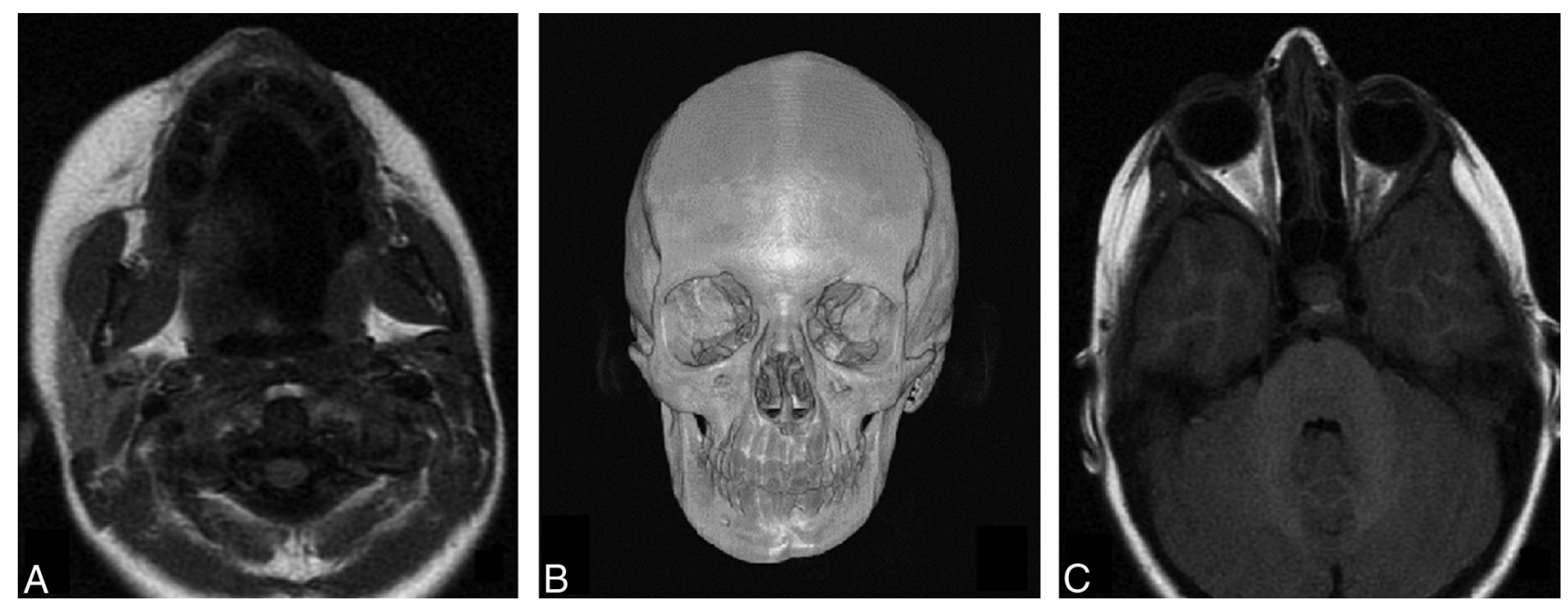

FIG 2. A 14-year-old boy who presented with progressive atrophy of the left face. $A$, T1-weighted image shows striking paucity of fat in the left face. $B, 3 D$ reconstruction of a $C T$ series demonstrates left facial hemiatrophy, particularly involving the mandible. $C$, T1-weighted image at the level of the orbits reveals left enophthalmos.

cles of mastication (Fig 2A). Skeletal findings were most pronounced in the mandible (Fig 2B). Enophthalmos of the left globe was present with relative paucity of retrobulbar fat (Fig $2 C)$. No intracranial imaging findings were observed at the time of presentation.

Case 3. A 24-year-old man with progressive hemiatrophy of the left lower face and left facial nerve dysfunction during the past 3 years presented for imaging evaluation of symptoms. MR imaging demonstrated near-absence of subcutaneous fat involving the inframaxillary face extending inferiorly to the visualized portions of the suprahyoid neck (Fig $3 A$ ). Only minimal asymmetry was noted involving the muscles and skeletal structures (Fig 3B). Involved tissues demonstrated normal T1 and T2 signal without pathologic contrast enhancement (Fig 3C). Limited evaluation of intracranial content revealed no abnormality. Diagnosis of PRS was made, given imaging findings and compatible clinical history and examination.

Case 4. An 11-year-old girl with a known diagnosis of PRS of unknown duration was experiencing increasing dermal sensi- tivity and tightness of the left hemiface and occasional pain along the left temple for the past year. CT revealed soft-tissue atrophy and osseous asymmetry most prominently in the left maxilla (Fig $4 A$ ), with extension to the left frontal scalp superiorly and left mandible inferiorly. There was deviation of the nose toward the side of atrophy (Fig $4 B$ ). The maxillary sinus, ethmoidal air cells, mandible, and osseous orbit were relatively smaller on the left (Fig 4C). No dedicated intracranial imaging was performed.

Case 5. A 30-year-old woman with known PRS presented with weakness and recurrent headaches and underwent MR imaging of the brain. Subtle thinning of the subcutaneous skin and fat was seen along the right scalp (Fig 5A). On FLAIR and T2-weighted images, increased signal was present in the ipsilateral subinsular, temporal, and occipital lobe white matter and in the cerebral peduncle (Fig $5 B,-C$ ). Susceptibility-weighted imaging demonstrated innumerable punctate foci throughout the right temporal, frontal, and occipital lobes and in the cerebellum (Fig 5D). The left hemisphere was normal. 

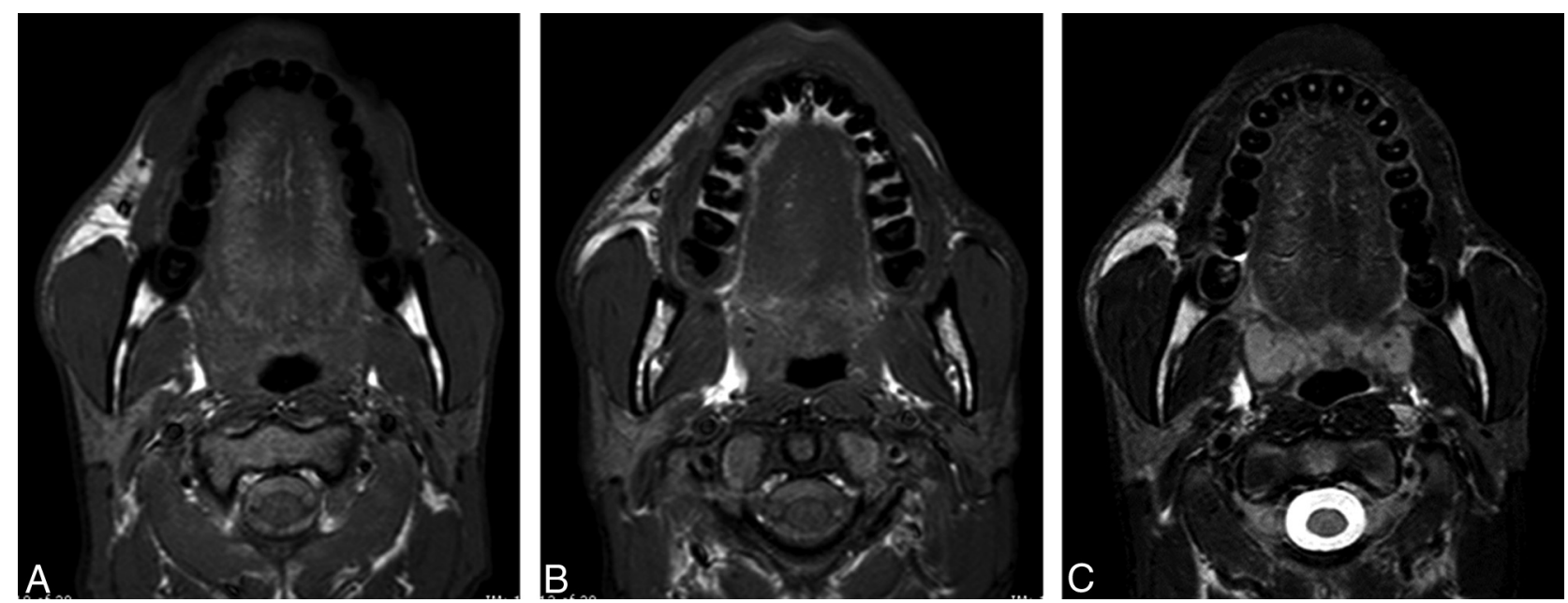

FIG 3. A 24-year-old man with progressive atrophy of the left face. $A$ and $B$, Axial T1-weighted MR images demonstrate left hemifacial atrophy primarily involving the skin and subcutaneous fat; only subtle asymmetry of the masseter is noted. $C$, T2-weighted image at the same level demonstrates normal T2 signal in the affected structures.
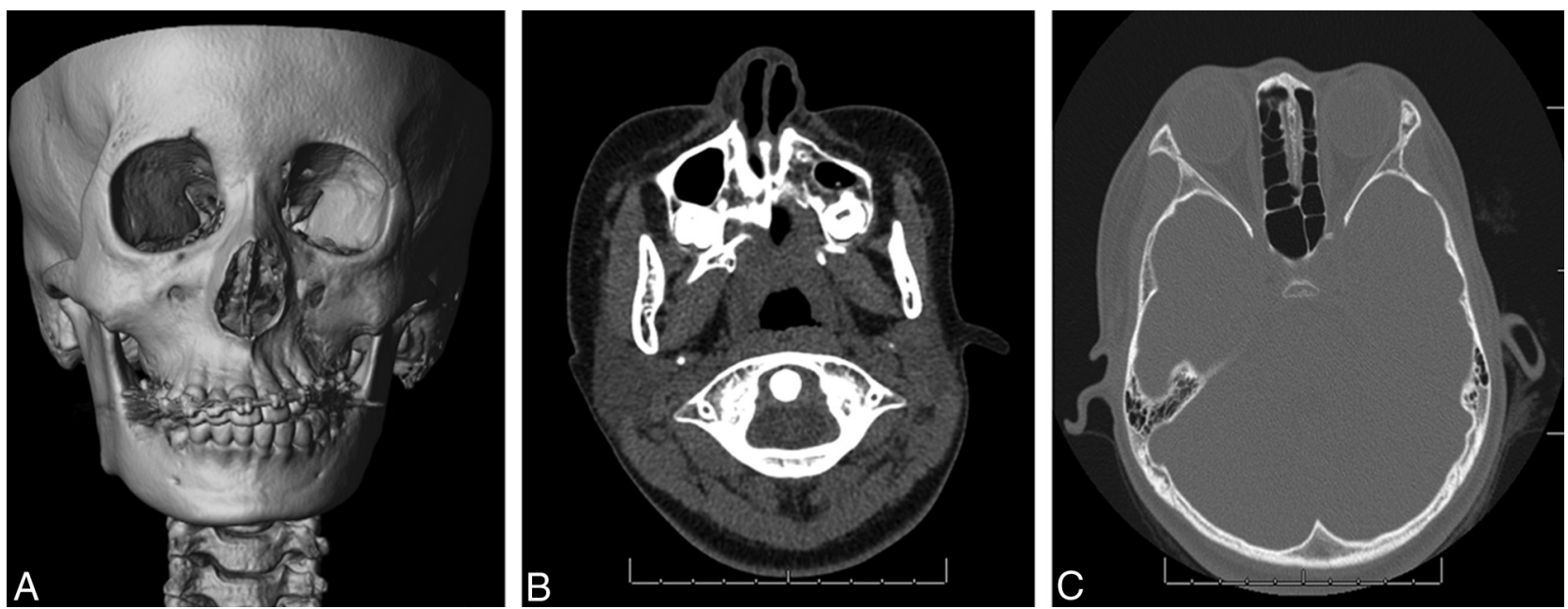

FIG 4. An 11-year-old girl with left-face sensitivity and left-temple pain. $A, 3 D$ reconstructions of a CT series show osseous asymmetry, most prominently in the left maxilla. B, Axial CT image at soft-tissue windows demonstrates deviation of the nose toward the affected side. C, Axial bone window demonstrates marked asymmetry in the volume of ethmoidal air cells.

Case 6. A 35-year-old woman with known left PRS presented with headache and weakness. MR imaging demonstrated increased T2 signal in the left hemispheric white matter. Involvement was geographic, with regional areas of confluent periventricular, deep, and subcortical white matter hyperintensity (Fig 6). The right hemisphere demonstrated only nonspecific punctate foci of white matter hyperintensity.

Case 7. A 68-year-old man with known PRS presented with clinical acute right middle cerebral artery occlusion. Noncontrast head CT demonstrated marked thinning of the left frontal scalp similar to an en coup de sabre deformity (Fig 7A). MR imaging demonstrated findings consistent with right MCA acute infarction (not shown). The left hemisphere was markedly abnormal with extensive white matter signal abnormality (Fig 7B). Asymmetric left ventriculomegaly and a relatively small ipsilateral sulcal size (Fig 4C) were present and suggested hydrocephalus, but their etiology remained unexplained.

\section{DISCUSSION}

Parry Romberg syndrome is a sporadic and rare condition that has been reported to be more common in females, ${ }^{2-6,10,15-17,20}$ without apparent geographic or ethnic predilection. ${ }^{15}$ Onset typically occurs during the first and second decades of life, resulting in an initially insidious but progressive hemiatrophy of the face during a span of 2-20 years, with a slight propensity for the left side. ${ }^{3-6,8-10,13-17,20,25}$ Seemingly without cause, the progression abruptly arrests and stabilizes, reaching a "burned-

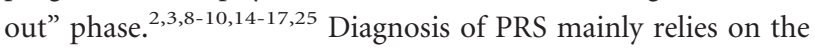
clinical history and examination and exclusion of other possibilities, supported by histopathologic and imaging studies. ${ }^{16}$

Patients characteristically experience atrophy of the skin and subcutaneous tissues and may develop atrophy of the underlying muscular, cartilaginous, osseous, and glandular structures as the disease progresses. ${ }^{1-9,13-17,20}$ The affected area typically begins in the maxillary or periorbital region and may expand to involve the forehead, perioral region, teeth, jaw, and neck to varying de- 

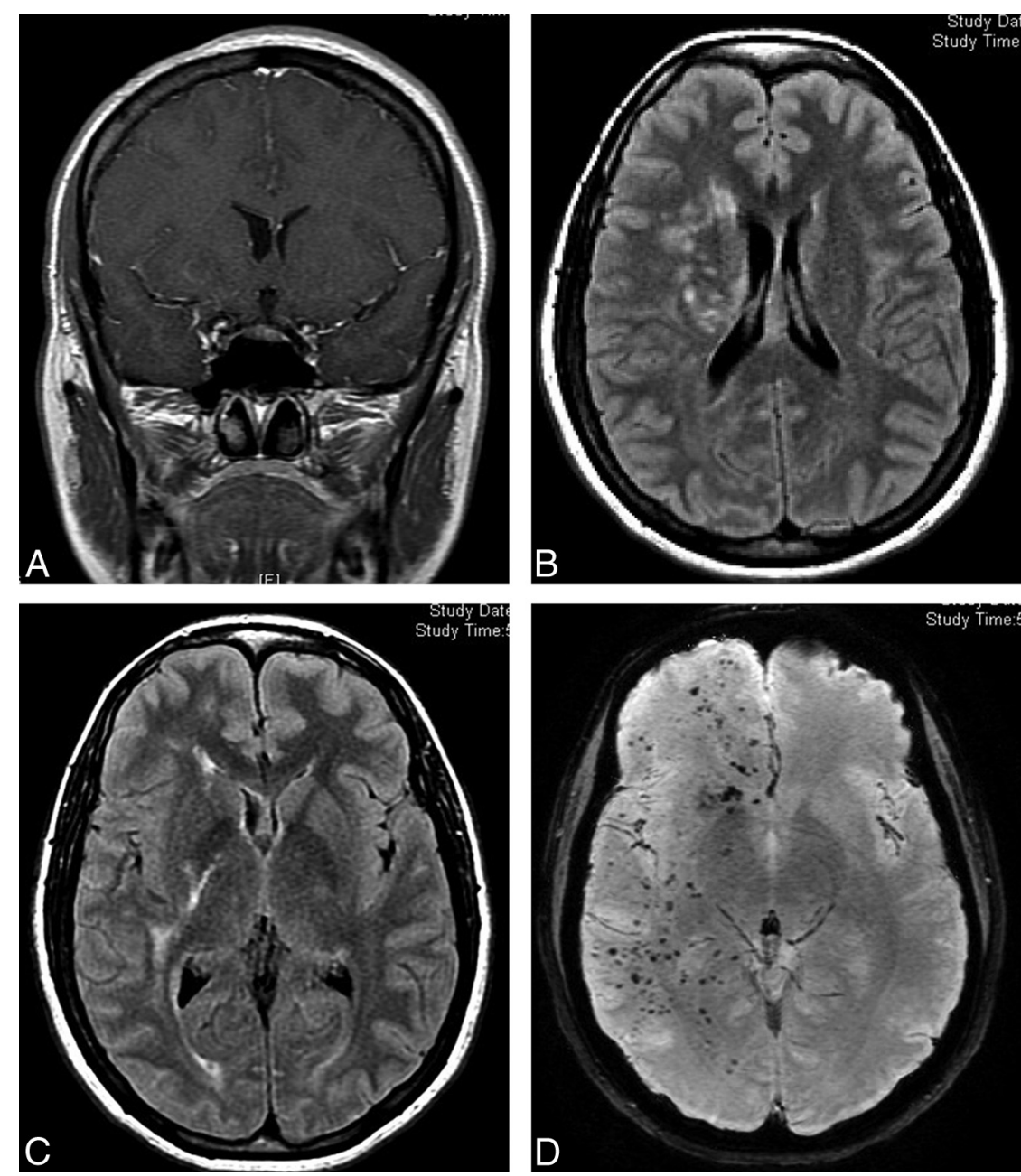

FIG 5. A 30-year-old woman with a history of PRS who presented for MR imaging evaluation for weakness and recurrent headaches. A, Coronal enhanced T1-weighted MR image demonstrates mild asymmetry of scalp thickness with relative paucity of subcutaneous fat on the right. $B$ and $C$, Axial FLAIR images demonstrate abnormal hyperintensity of the white matter in the right corona radiata, internal capsule, and temporal region. $D$, Axial susceptibilityweighted image demonstrates innumerable punctate foci of susceptibility throughout the right cerebral hemisphere.
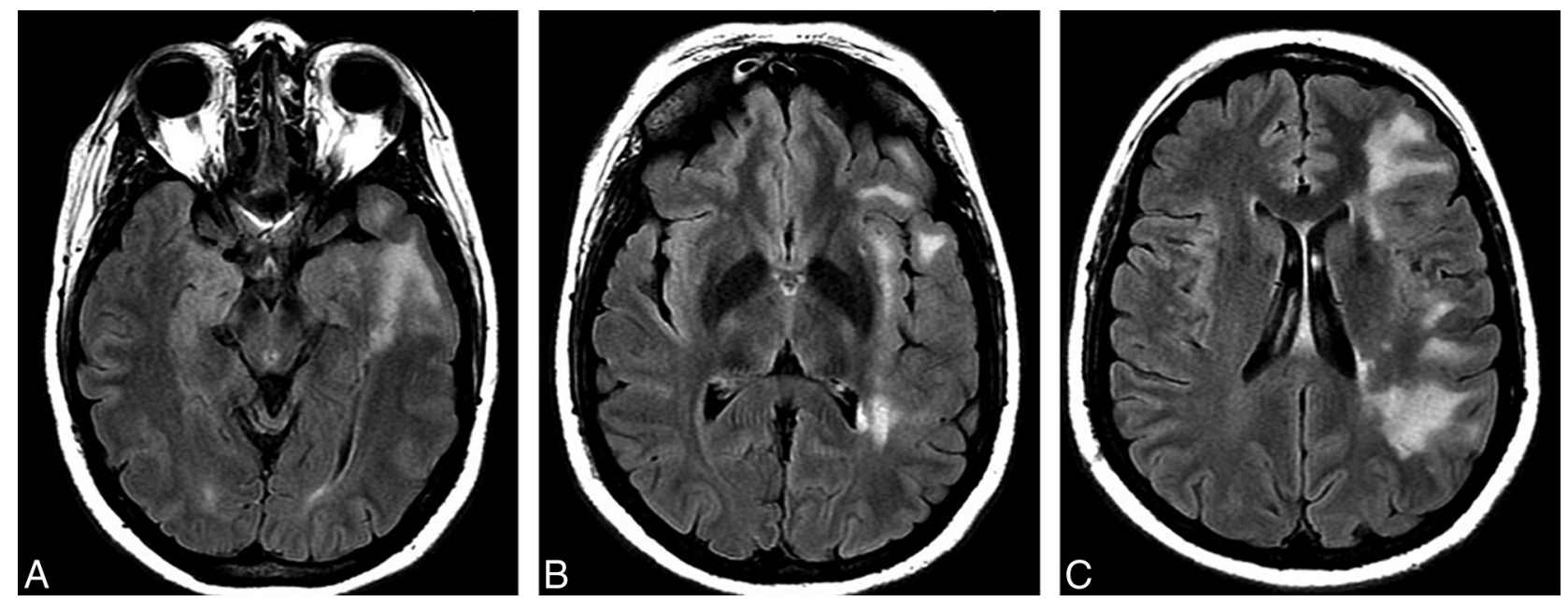

FIG 6. A 35-year-old woman with a history of PRS presented for MR imaging evaluation for headaches and weakness. Axial FLAIR images demonstrate hyperinstensity in the left cerebral periventricular to subcortical white matter. The pattern of signal abnormality is geographic, with subtotal involvement of frontal, parietal, and temporal lobes. 

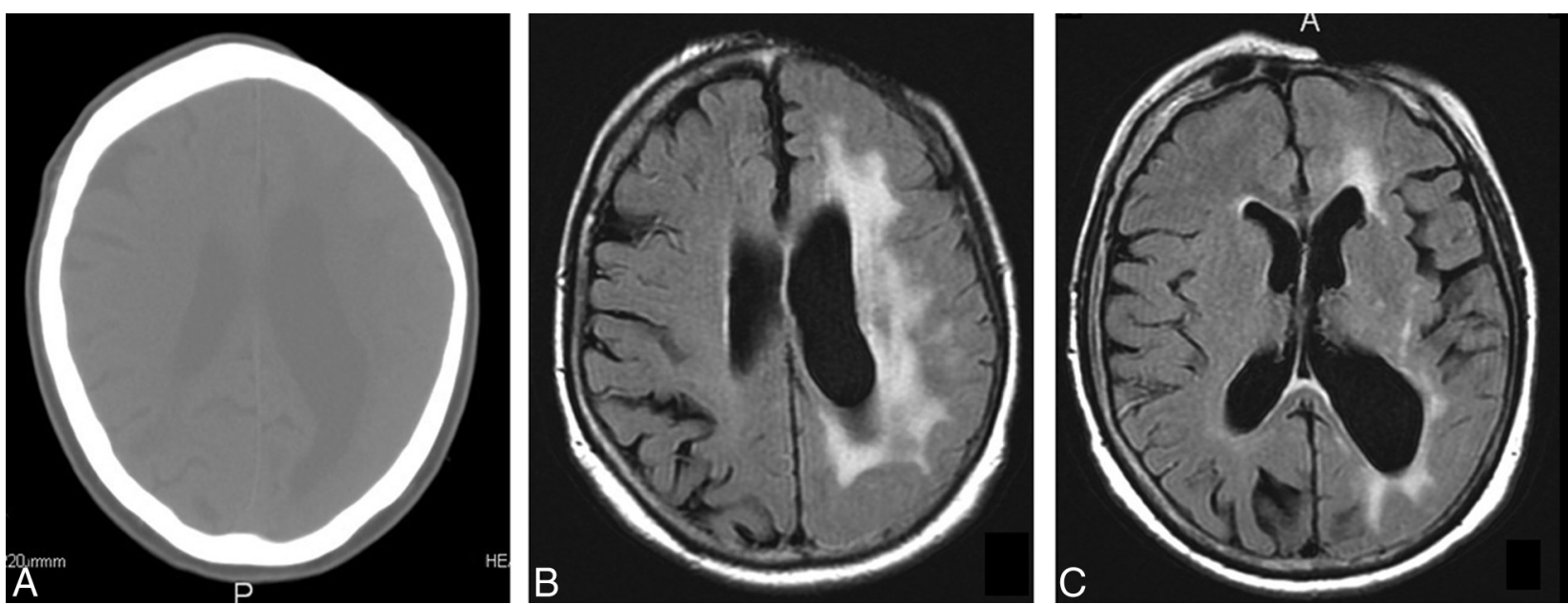

FIG 7. A 68-year-old man with PRS who presented with acute right MCA infarct. A, Axial CT image demonstrates striking focal loss of scalp thickness in the left frontal region. $B$, Axial FLAIR images demonstrate confluent signal abnormality in the high left frontal and parietal white matter. C, Etiology of left ventriculomegaly and sulcal asymmetry was uncertain.

pathophysiology remains limited. ${ }^{3}$ Proposed etiologies have included trauma, genetic predisposition, infection, radiation exposure, embryonic developmental dysfunction, sympathetic cervical ganglion dysfunction, and metabolic and endocrinologic disturbances. ${ }^{1-3,5-8,13-17}$ Currently, the strongest laboratory and histologic evidence supports an inflammatory autoimmune disorder with or without associated vasculopathy. ${ }^{1-6,12,14,15,17}$ Clinical improvement with immunosuppressive therapy during active disease further supports an underlying immunologic-mediated process. $^{8,25}$

On radiologic assessment, common facial imaging findings include varying degrees of hemiatrophy accompanied by obliteration of fat planes, ipsilateral deviation of the aerodigestive tract, and enophthalmos due to loss of retrobulbar fat. ${ }^{15,17}$ No abnormal CT attenuation or MR signal has been reported within the affected facial structures. ${ }^{15}$ Intracranially, the most common findings are the following: 1) ipsilateral linear or discrete subcortical calcifications in the frontal lobe, 2) white matter hypoattentuation on CT corresponding to hyperintense T2 signal on MR imaging, ${ }^{1-3,7-10,13-18}$ and 3) ipsilateral focal or hemispheric brain atrophy. ${ }^{16-18}$ These findings typically occur ipsilateral to the affected side of the face ${ }^{15}$ and may worsen with disease progression. ${ }^{1}$ Less frequently described imaging findings include the loss of cortical gyration, cortical thickening, ventricular dilation, focal corpus callosum infarcts, leptomeningeal thickening and contrast enhancement, attenuated mineral deposits, hamartomas, and MR signal abnormality involving the thalamus or gray matter. 2,3,7,13,15-17 Vascular abnormalities such as microhemorrhages, malformations, stenoses, and aneurysms have also been reported in association with PRS. ${ }^{3,13,15-17,25}$

In our case series, we demonstrated a variety of imaging findings in 7 patients with PRS. All patients had varying degrees of facial atrophy, and the affected extracranial structures retained normal signal patterns on MR imaging. Our first 4 patients underwent scanning for evaluation of facial findings. In 1 of the 4 patients, additional brain imaging was performed but revealed no abnormality. The remaining 3 patients with known diagnoses of PRS underwent dedicated brain imaging to evaluate neurologic symptoms; all had substantial intracranial manifestations with white matter signal abnormality ipsilateral to the side of facial atrophy being a consistent finding. One patient demonstrated a striking pattern of innumerable foci of susceptibility in the ipsilateral brain - these may represent microhemorrhages or foci of mineralization. In another patient, unexplained ventriculomegaly and associated small sulci could represent underlying hydrocephalus or may reflect an as-yet-undocumented intracranial manifestation of PRS.

PRS shares many clinical features, such as age of onset, associated neurologic symptoms, and cutaneous presentations with en coup de sabre, a rare variant of linear scleroderma that occurs along the anterior aspect of the scalp. ${ }^{10,25,26}$ These 2 entities are commonly discussed in conjunction due to their overlapping presentations and lack of distinguishing clinical features. ${ }^{3,5,8,10,17,25}$ To make differentiation even more complicated, conversion of en coup de sabre to PRS has been reported, ${ }^{7,17,21}$ and 30\%$40 \%$ of patients with PRS have changes typical of scleroderma localized outside the face. ${ }^{5,8,12}$ This has led many authors to consider these 2 entities variants that share a common underlying pathogenesis related to linear scleroderma. ${ }^{17-19,21,22,25}$ Other authors, however, argue against this claim ${ }^{10,26}$ because deeper head and neck structures, such as the oral or pharyngeal musculature, are not commonly involved in en coup de sabre, and there are typically elevated titers of autoantibodies in en coup de sabre but not in PRS. ${ }^{21,22}$ On the other hand, PRS demonstrates little-to-no sclerosis of the cutaneous and subcutaneous structures, variable perivascular chronic inflammation, and preservation of dermal elastic tissue on histopathologic examinations, differentiating it from the spectrum of scleroderma diseases. ${ }^{3,16,17,25,26}$

PRS also shares features with Rasmussen encephalitis, an autoimmune/inflammatory disorder affecting 1 side of the brain, resulting in refractory epilepsy and progressive hemiplegia and typically affecting children in the first decade of life. ${ }^{14,27,28}$ On imaging, Rasmussen encephalitis is characterized by predominately unilateral hemispheric areas of hyperintense T2 signal with eventual development of unilateral ce- 
rebral atrophy, ${ }^{27,28}$ findings that may be indistinguishable from intracranial imaging features of PRS. There have been reported cases of coexisting Rasmussen encephalitis and PRS in pediatric patients, ${ }^{4,8,14,15,17}$ supporting a possible pathophysiologic correlation between these 2 entities. In younger patients, differentiating these 2 entities may be difficult, but clinical signs and symptoms, such as the presence of epilepsia partialis continua, a hallmark symptom of Rasmussen encephalitis, and involvement of cutaneous structures should guide the clinician and radiologist to the correct diagnosis. ${ }^{1,4,7,8,27,28}$

Other diseases in which facial asymmetry is a prominent clinical feature include hemifacial microsomia (first and second brachial arch syndrome) and Goldenhar syndrome, but unlike PRS, these conditions are typically congenital and nonprogressive. ${ }^{19,21,22}$ Hemifacial hyperplasia causes asymmetry of the face as well, but rather than atrophy, this entity is characterized by overgrowth and hyperplasia. ${ }^{21,29}$ Partial lipodystrophy (Barraque-Simons syndrome) may have manifestations similar to those of PRS, but typically these are bilateral. ${ }^{19,22}$ Silent sinus syndrome may also be considered, but these patients present later in life with characteristic imaging findings of opacification and atelectasis of the maxillary sinus and evidence of ipsilateral ostiomeatal unit obstruction. ${ }^{30}$

No standard treatment algorithm currently exists for PRS, ${ }^{12,17}$ especially given that response to treatment is difficult to assess. ${ }^{15}$ In general, treatment goals are to obtain seizure control if seizures are present, provide symptomatic relief, and halt disease progression. ${ }^{17,18}$ PRS-related seizures are usually treated with anticonvulsive therapy despite their commonly refractory nature. ${ }^{1,4,14} \mathrm{At}$ times, lobectomy may be warranted for seizure control. ${ }^{4,8}$ Additionally, similar to treatments for scleroderma, immunosuppressive therapies, ranging from topical corticosteroids to systemic corticosteroids, immunomodulators, and plasmapheresis, have been used with varying degrees of success. Antimalarials, antibiotics, vitamin $\mathrm{D}_{3}$ analogues, and penicillamine are also treatment options that have demonstrated variable responses. ${ }^{5,8,10,14-18}$ Once the disease stabilizes, cosmetic therapies, including pulse dye lasers, dermal fat grafts, autologous fat grafts, muscle flap grafts, free silicone injections, and bone augmentations, are available for aesthetic management. ${ }^{10,23,24}$ Recently, autologous fat grafting with adipose-derived stem cells has been shown to provide the most favorable cosmetic results. ${ }^{23,24}$ In these incidences, objective serial volumetric analysis of both hemifaces is important to monitor fat reabsorption, an indicator of successful grafting. Volumetric calculations can be easily achieved with 3D reconstructions of CT data acquired periprocedurally and on serial follow-up examinations, but alternative methods are being investigated as well due to concerns for cost and patient radiation exposure. $^{23,24}$

In summary, PRS is a rare, self-limiting, and slowly progressive hemiatrophy of the face that typically affects the skin and subcutaneous tissues and may affect deeper tissues such as the musculature, cartilage, and osseous structures. Neurologic and ophthalmologic symptoms are common, but underlying pathophysiology remains uncertain. Intracranial involvement is best evaluated with MR imaging and can range widely, with the most common findings being parenchymal calcifications, white matter abnormalities, and brain atrophy. In addition to evaluating the extent of disease, radiologic assessments may also facilitate the exclusion of other differential considerations, help monitor disease progression, and evaluate posttreatment responses.

\section{REFERENCES}

1. Yano T, Sawaishi Y, Toyono M, et al. Progressive facial hemiatrophy after epileptic seizures. Pediatr Neurol 2000;23:164-66

2. Aynaci FM, Sen Y, Erdol H, et al. Parry-Romberg syndrome associated with Adie's pupil and radiologic findings. Pediatr Neurol 2001;25:416-18

3. Pichiecchio A, Uggetti C, Egitto MG, et al. Parry-Romberg syndrome with migraine and intracranial aneurysm. Neurology 2002;59:606-08

4. Shah JR, Juhasz C, Kupsky WJ, et al. Rasmussen encephalitis associated with Parry-Romberg syndrome. Neurology 2003;61:395-97

5. Stone J. Parry-Romberg syndrome: a global survey of 205 patients using the internet. Neurology 2003;61:674-76

6. Moko SB, Mistry Y, Blandin de Chalain TM. Parry-Romberg syndrome: intracranial MRI appearances. J Craniomaxillofac Surg 2003;31:321-24

7. Sommer A, Gambichler T, Bacharach-Buhles M, et al. Clinical and serological characteristics of progressive facial hemiatrophy: a case series of 12 patients. J Am Acad of Dermatol 2006;54:227-33

8. Paprocka J, Jamroz E, Adamek D, et al. Difficulties in differentiation of Parry-Romberg syndrome, unilateral facial sclerodermia, and Rasmussen syndrome. Childs Nerv Syst 2006;22:409-15

9. Okumura A, Ikuta T, Tsuhi T, et al. Parry-Romberg syndrome with a clinically silent white matter lesion. AJNR Am J Neuroradiol 2006;27:1729-31

10. Tollefson MM, Witman PM. En coup de sabre morphea and ParryRomberg syndrome: a retrospective review of 54 patients. $J \mathrm{Am}$ Acad of Dermatol 2007;56:257-63

11. Restivo DA, Milone P. Teaching neuroimages: progressive facial hemiatrophy (Parry-Romberg syndrome) with ipsilateral cerebral hemiatrophy. Neurology 2010;74:e11

12. Lazaridou E, Giannopoulou C, Apalla Zoi, et al. Parry-Romberg syndrome. J Dermatol Case Rep 2010;4:30-32

13. Blitstein MK, Vecchione MJ, Tung GA. Parry-Romberg syndrome. Applied Radiol 2011;40:34-36

14. Longo D, Paonessa A, Specchio N, et al. Parry-Romberg syndrome and Rasmussen encephalitis: possible association: clinical and neuroimaging features. J Neuroimaging 2011;21:188-93

15. Sharma M, Bharatha A, Antonyshyn OM, et al. Case 178: Parry-Romberg syndrome. Radiology 2012;262:721-25

16. Madasamy R. Jayanandan M, Adhavan UR, et al. Parry Romberg syndrome: a case report and discussion. J Oral Maxillofac Pathol 2012;16:406-10

17. El-Kehdy J, Abbas O, Rubeiz N. A review of Parry-Romberg syndrome. J Am Acad of Dermatol 2012;67:769-84

18. Chiu YE, Vora S, Kwon EK, et al. A significant proportion of children with morphea en coup de sabre and Parry-Romberg syndrome have neuroimaging findings. Pediatr Dermatol 2012;29:738-48

19. Vykuntaraju KN, Sahana, Shivananda. Parry-Romberg syndrome. Indian Pediatr 2013;50:255

20. Dalla Costa G, Colombo B, Libera DD, et al. Parry Romberg syndrome associated with chronic facial pain. J Clin Neurosci 2013;20:1320-22

21. Khan M, Khan M, Negi R, et al. PRS with localized scleroderma: a case report. J Clin Exp Dent 2014;6:e313-16

22. Trisal D, Kumar N, Dembla G, et al. Parry-Romberg syndrome: uncommon but interesting. BMJ Case Rep 2014;pii:bcr2013201969

23. Koh KS, Oh TS, Kim H, et al. Clinical application of human adipose tissue-derived mesenchymal stem cells in progressive hemifacial atrophy (Parry-Romberg disease) with microfat grafting techniques using 3-dimensional computed tomography and 3-dimensional camera. Ann Plast Surg 2012;69:331-37 
24. Chang Q, Li J, Dong Z, et al. Quantitative volumetric analysis of progressive hemifacial atrophy corrected using stromal vascular fraction-supplemented autologous fat grafts. Dermtol Surg 2013; 39:1465-73

25. Kister I, Inglese M, Laxer RM, et al. Neurologic manifestations of localized scleroderma: a case report and literature review. Neurology 2008;71:1538-45

26. Miller K, Lehrhoff S, Fischer M, et al. Linear morphea of the forehead (en coup de sabre). Dermatol Online J 2012;18:22

27. Geller E, Faerber EN, Legido A, et al. Rasmussen encephalitis: com- plementary role of multitechnique neuroimaging. AJNR Am J Neuroradiol 1998; 19:445-49

28. Faria AV, Reis F, Dabus GC, et al. MRI findings in the diagnosis and monitoring of Rasmussen's encephalitis. Arg Neuropsiquiatr 2009; 67:792-97

29. Bou-Haidar P, Taub P, Som P. Hemifacial lipomatosis, a possible subtype of partial hemifacial hyperplasia: CT and MR imaging findings. AJNR Am J Neuroradiol 2010;31:891-93

30. Hourany R, Aygun N, Della Santina CC, et al. Silent sinus syndrome: an acquired condition. AJNR Am J Neuroradiol 2005;26:2390-92 